Supplement of Biogeosciences, 14, 2151-2154, 2017

http://www.biogeosciences.net/14/2151/2017/

doi:10.5194/bg-14-2151-2017-supplement

(C) Author(s) 2017. CC Attribution 3.0 License.

(c) (i)

Supplement of

\title{
Technical note: an economical apparatus for the observation and harvest of mineral precipitation experiments with light microscopy
}

Chris H. Crosby and Jake V. Bailey

Correspondence to: Chris H. Crosby (crosb118@umn.edu)

The copyright of individual parts of the supplement might differ from the CC-BY 3.0 licence. 
Diffusion gel material and solution concentrations can be altered as required for different experiments, but the conditions under which this apparatus was developed, and those used to produce figure 1E, are as follows:

gelatin: type $\mathrm{A}, 1 \mathrm{~g} / \mathrm{ml}$ water, $\mathrm{pH} \sim 4$

5 cation solution: $0.133 \mathrm{M} \mathrm{Ca}^{2+}\left[\mathrm{CaCl}_{2} \cdot 2 \mathrm{H}_{2} \mathrm{O}, \mathrm{pH} \sim 8\right]$

anion solution: $0.08 \underline{\mathrm{M}}\left(\mathrm{PO}_{4}\right)^{3-}\left[\mathrm{NaHPO}_{4}, \mathrm{pH} \sim 8\right]$

$\square$ anion solution: $0.027 \underline{\mathrm{M} \mathrm{F}^{-}}\left[\mathrm{KF} \cdot 2 \mathrm{H}_{2} \mathrm{O}, \mathrm{pH} \sim 8\right]$

Nascent precipitation is observed within $\sim 30$ hours.

\section{S1 Supplement A: Setup block \& adaptor construction}

Note: The width and length of the setup block and adaptor-spacer should be slightly smaller than the long coverslip. The dimensions shown in Fig. 1 assume a standard long coverslip size of 24 x $60 \mathrm{~mm}$. The bore diameter should be $\sim 3-4 \mathrm{~mm}$ less than a side of the square coverslip. The bore size shown in Fig. 1 assumes an 18 x 18 mm square coverslip. Revise the

15 dimensions as required to accommodate differently sized cover slips.

\section{S1.1 Setup block}

Machine the setup block as indicated in Fig. 1a.

\section{S1.2 Adaptor-spacer}

\section{S1.2.1 Material needed}

$\square$ two clear, rigid flat surfaces ("sheets") such as $8 \times 10$ in. acrylic or glass sheets

$\square$ two microscope slides (of same thickness)

$25 \square$ one square coverslip

$\square$ silicone epoxy molding material (Castin'Craft ${ }^{\mathbb{R}}$ EasyMold silicone putty, or similar)

$\square$ cutting blade, such as an X-acto ${ }_{\circledast}$ blade or similar

$\square$ hole punch, $\sim 16 \mathrm{~mm}$ or $\sim 5 / 8$-in.

$30 \quad$ S1.2.2 Molding procedure

$\square$ Position a square coverslip and two slides on one of the sheets as shown in Fig. S1a. The slides will serve as spacers to set the thickness of the adaptor-spacer and their positioning isn't critical, but they should be at least $\sim 40 \mathrm{~mm}$ apart. The square coverslip will form an indent in the adaptor to accept a square coverslip in the assembled unit.

$\square$ Mix a small volume of silicone epoxy, per instructions.

$35-$ Form the epoxy into a cylinder and gently press it over the coverslip between the slides, avoiding air bubbles under the epoxy.

Place the second sheet on the epoxy and press the epoxy into a sheet the thickness of the slides, again observing to avoid air bubbles between the sheets and the epoxy.

Allow epoxy to set, per instructions.

$40-$ Remove the top sheet, pick up the molded epoxy and carefully remove the square coverslip.

$\square$ Put the epoxy, indentation side up, back on the sheet and cut out a slot, as shown in Fig. S1b(a).

Insert a piece of paper into the hole punch as far as it will go without bending, and punch a hole in it Fig. S1b(b).

Mark off the distance from the center of the hole to the feed-edge of the paper. 
Mark off the distance from the center of the hole to the feed edge of the paper.

Using this distance, trim one length of the adaptor-spacer so that when it has been fully inserted into the punch a hole will be punched directly in the center of the indent as shown in Fig. S1b(c).

$\square$ Insert the adaptor-spacer into the hole punch, aligned side-to-side so that the hole will be punched in the center of the indentation Fig. S1b(d). (Practice positioning a sheet of paper in the hole punch before punching the adaptor.)

$\square$ Trim the adaptor to the size of the setup block Fig. S1b(e).

$\square$ Check the position of the hole and slot by holding it against a setup block.

\section{S2 Supplement B: Preassembly preparation}

\section{S2.1 Preparing for experimental sterility}

To assure a controlled, sterile experiment, all objects and solutions in contact with the gel should be sterilized. Either autoclave them ahead of assembly, or sterilize them in $70 \%$ ethanol, as mentioned in $\S 3.2$, below.

\section{S2.2 Addition of secondary organics}

Secondary organic material (an organic substrate other than the gel material) can be immobilized before assembly of the setup. Place the material in the center of the long coverslip and immobilize/adhere it by allowing it to air dry or quickly passing it over a flame. Rinse as needed to remove media precipitates. Staining of organics may also be accommodated.

\section{S2.3 Focusing aids (optional)}

$\square$ Holding a square cover slip securely against a flat surface, carefully scribe a small ' $L$ ' extending from the middle of one side. Rinse off glass scrapings. The combined position and direction of the $\mathrm{L}$ will indicate which side is up - the scribed surface will be positioned against the gel and can be used to locate the 'top' surface of the gel layer under the microscope.

$\square$ Scribe another ' $\mathrm{L}$ ' in the long cover slip parallel to the length, $\sim 5-6 \mathrm{~mm}$ from the edge to indicate the 'bottom' outer surface of the gel layer. Rinse off glass scrapings. In the finished setup, these two scribe marks should overlap near the outer area of the gel, with the scribed surfaces both in contact with the gel surface. 


\section{$\underline{\text { S3 Supplement C: Assembling and activating the apparatus }}$}

Note: The steps listed here assume the use of gelatin as the primary organic polymer (the diffusion gel.) In developing this apparatus and protocol, gelatin was chosen as a diffusion gel because its gel is transparent and amenable to optical observation and imaging, and because it is soluble in hot water. It is intended that experimental details will represent

5 conditions as desired by the experimenter, such as use of a different diffusion polymer. For work in which a different diffusion material is to be used, if extraction of precipitates is desired it will be important to consider both the solubility requirements of the diffusion material and the stability of precipitates and precursor molecules under whichever extraction method is required.

Ion solutions can be changed mid-experiment to alter solution chemistry, $\mathrm{pH}$, Eh, etc. as desired. Addition to and/or

10 removal and replacement of the initial ion solution(s) can be done under sterile conditions in a biological hood (see § S3.7, below.)

Some of these assembly steps are labelled "skill steps." Best results are attained with practice before attempting to assemble and activate a live setup. Components are joined by a layer of Vaseline ${ }^{\mathrm{TM}}$. If Vaseline doesn't stand up to your handling, thicken it by adding small amounts of paraffin and lanolin and melting them together. Paraffin will cause the

15 mixture to set more solidly, but too much paraffin may cause it to set so quickly that you will have to work fast, and may make the material brittle and more prone to fracture during experiment handling. Alternatively, a dab of candle wax applied to each end of the assembly (Fig. 1d) can immobilize the setup block-adaptor-long cover slip assembly.

S3.1 Materials required for assembly [Assumes gelatin as primary polymer.]

20 setup components

$\square$ suction tool, such as a Model Pal ${ }^{\mathrm{TM}}$ Suction Handling Tool, or similar

$\square$ hot plate with magnetic stir capacity

$\square$ small stir bar

$70 \%$ ethanol in a small container for sterilizing various parts and tools

$25 \square$ small beaker for mixing gelatin

$\square$ gelatin powder

$\square \geq 10 \mathrm{~mL}$ nanopure or sterile water, for gelatin

- Vaseline ${ }_{\circledast}$

2 of: 2 x 3-inch glass slide, or similar

$30 \quad 3$ of: $3 \mathrm{~mL}$ syringes with needles

$\square$ disposable pipette

$\square$ pointed tweezers

$\checkmark$ sharp small blade

$\square$ fine metal probe

$35 \square$ cation solution

$\square$ anion solution

small wax candle

$\square \sim 10 \mathrm{~mL}$ sterile water, for testing watertightness 
S3.2 Initial steps: mix gel [Assumes gelatin as diffusion gel. Revise as needed for a different gel.]

$\square$ Before turning the hot plate on, clean its surface, sterilize it with $70 \%$ ethanol and allow it to dry.

$\square$ Place items that have not been autoclaved or sterilized (the stir bar, tweezers, probe tip, coverslips, two 2 × 3 -inch slides, an adaptor-spacer \& setup block, etc.) in a small container of $70 \%$ ethanol to sterilize them. Before using each of them in the following steps, remove them from the ethanol and allow them to dry.

$\square$ Turn hot plate to $\sim 30 \mathrm{C}$.

$\square$ Measure the nanopure water for the gel and heat it in the small beaker.

$\square$ Measure out the gel powder, add it to the heated water, add the small stir bar and mix.

$\square$ Dip the suction tool tip into the ethanol to sterilize it and use it to transfer the square coverslip, adaptor-spacer \& setup block to the hot plate. Move the $2 \times 3$-inch slides to the bench top, and place the long coverslip on one of them.

$\square$ Wipe a thin even layer of Vaseline ${ }_{\circledast}$ onto bottom (flat) surface of the adaptor-spacer.

$\square$ Center the adaptor onto the long coverslip and press them together.

Verify the seal by the appearance of the adapter material against the long coverslip. Air bubbles should look lighter or darker than well sealed areas and should be avoided where they could allow leakage.

$\square$ Check the mixing gel and slow the stir rod to release any air bubbles entrained in it.

\section{S3.3 Add gel [skill step]}

Apply Vaseline along the edges of the recessed portion of the adaptor-spacer, avoiding the channels and the gel.

$\square$ [skill step] Pull $\sim 1 \mathrm{~mL}$ of gel into one of the syringes, minimizing entrained air bubbles (pull the syringe plunger slowly, release the plunger and allow it to stop moving before removing the needle tip from the gel.)

$\square$ [skill step] Extrude a small volume of gel into the center bore to a slight convex meniscus.

Immediately remove bubbles from the gel as needed, using the tweezers in a horizontal 'cutting' motion to pick up and remove bubbles.

\section{$25 \quad$ S3.4 Add small cover slip}

$\square$ Check that the Vaseline along the edges of the recessed portion hasn't been rubbed off.

[skill step] Use the suction tool to pick up the square coverslip and press it over the gel and into the recessed portion of the adaptor-spacer. The gel should fill the volume beneath the square coverslip and a small volume of gel should push out the adaptor-spacer channels.

$30 \square$ Check that the square coverslip is sealed against the Vaseline ${ }_{\circledast}$ on the adaptor.

$\square$ [skill step] Verify that the channels are both clear of Vaseline $\mathbb{B}_{\mathfrak{B}}$. If needed, use a fine probe to clear them of any Vaseline $\mathbb{B}_{\mathbb{B}}$. Gel intruding into the side bores is OK.

\section{S3.5 Add setup block}

35 Apply a layer of Vaseline ${ }_{\mathbb{B}}$ onto the bottom of the setup block.

$\square$ [skill step] Place the setup block on the assembly. Apply downward pressure (only) as needed to get a complete seal (sideward pressure will cause the components to slip out of alignment.) See that seal is complete and neither cover slip has broken.

40 the setup block side of each end of the long coverslip where it meets the setup block (Fig. 1d).

$\square$ Gently remove any extraneous Vaseline $e_{\circledast}$ from the exterior and place the unit on a clean surface.

\section{S3.6 Add ion solutions, seal side bores}

$\square$ Pipette sterile water into one of the side bores, wait and watch that: 1) no water leaks out of the unit and 2) no water leaks into the other bore. This can happen if the seal between the long coverslip and the setup block is not water tight. If there is leakage, repair the seal or start over. Once confident that there is no leakage pour the water out and dry the unit.

$\square$ Repeat for other side.

Add ion solutions to the side bores and label them.

Wick off any water on the setup block exterior. 
Cut a section of sterile bandage material, leaving the backing paper on. If using 2-3/8 x 2-3/4-inch $3 \mathrm{M} \mathrm{Nexcare}^{\mathrm{TM}}$

Tegaderm $^{\mathrm{TM}}$, cut it lengthwise and set one of the halves aside.

Remove the backing, stretch the bandage across the top of the three bores and press it onto the setup block to seal.

$\square$ Scotch Tape ${ }^{\mathrm{TM}}$ (or similar) across the length of the bandage to preclude evaporation through the bandage material.

5 Use a small sharp blade to cut the tape+Tegaderm ${ }^{\mathrm{TM}}$ away from the center bore.

$\square$ Recheck all seals.

The setup is now activated. Label the setup and place it on a paper towel or similar to detect leakages.

$\square$ Image as desired, handling carefully so as not to break the Vaseline ${ }_{\circledast}$ seals.

$10 \quad$ S3.7 Changing ion solutions mid-experiment [optional - perform under sterile conditions, such as a biological hood]

To keep the solution sterile, wipe a small amount of ethanol on the tape surface where it will be punctured.

Using a sterilized blade, carefully cut a small slit in the sealing material over one of the solution wells.

Use a sterile needle and syringe to remove the original solution.

$\square$ Use a different sterile needle and syringe to introduce a new solution.

15 Reseal with Tegaderm ${ }^{\mathrm{TM}}$ or similar sterile material.

Seal against evaporation with clear watertight tape as described in S3.6, above.

\section{S4 Supplement D: Harvesting procedure}

Note: Once the gel is exposed to air it will begin to harden, and chipping gel off coverslips may break them into slivers. The gel can be kept soft in cool water. When cutting portions of gel away from the coverslip, place the coverslip against a firm

flat surface.

\section{S4.1 Materials needed}

$\square$ thin X-actoтм blade or similar

$\square$ dissecting microscope

$30 \quad$ container for waste ion solutions

small container of cool DI water

small squeeze bottle of DI water

$\square$ beaker of hot DI water

$\square$ small $(\sim 5-25 \mu \mathrm{L})$ pipet and tips

$35 \square$ prepared SEM stubs or similar for follow-up analysis and/or imaging

\section{S4.2 Procedure}

Open/remove the tape over the ion solutions and pour the solutions into a disposal container.

$\square$ Working over a catch tray, separate the long coverslip from the adaptor-spacer by gently sliding a thin X-асtотм blade

40 between them and slowly applying a slight twisting pressure against the long coverslip. It should bend a bit and allow the seal to break. Hold the unit so it reflects overhead light to see where the seal is broken - it will be lighter than the intact seal.

Using the same procedure, remove the adaptor-spacer from the setup block. Only the square coverslip and gel plug should remain attached to adaptor-spacer. Leaving the gel plug attached to the cover slip will make it easier to handle. Place it on a firm even surface, such as the stage of a dissecting microscope.

Cut away the portions of interest and place them on two-sided carbon tape on an SEM stub or similar. 
$\square$ Rinse gel off the precipitates by repeatedly pipetting small amounts of hot sterile water onto the harvested precipitates, letting the gel melt, and then removing the melted gel + water. Repeat as needed to assure removal of gel from precipitates.

$\square$ For gels with smaller or more numerous precipitates: Place the gel plug in an epitube of hot water, immerse it in a hot water bath, shake it gently and hold it vertically in the hot water bath to allow precipitates to settle. Slowly remove supernatant by pipette. Repeat as needed to assure removal of gel from precipitates. Remove the final, rinsed precipitates + bottom rinse water from the epitube by pipette and place on a carbon-taped stub. Let the water evaporate and further rinse the precipitates on the stub as described above.

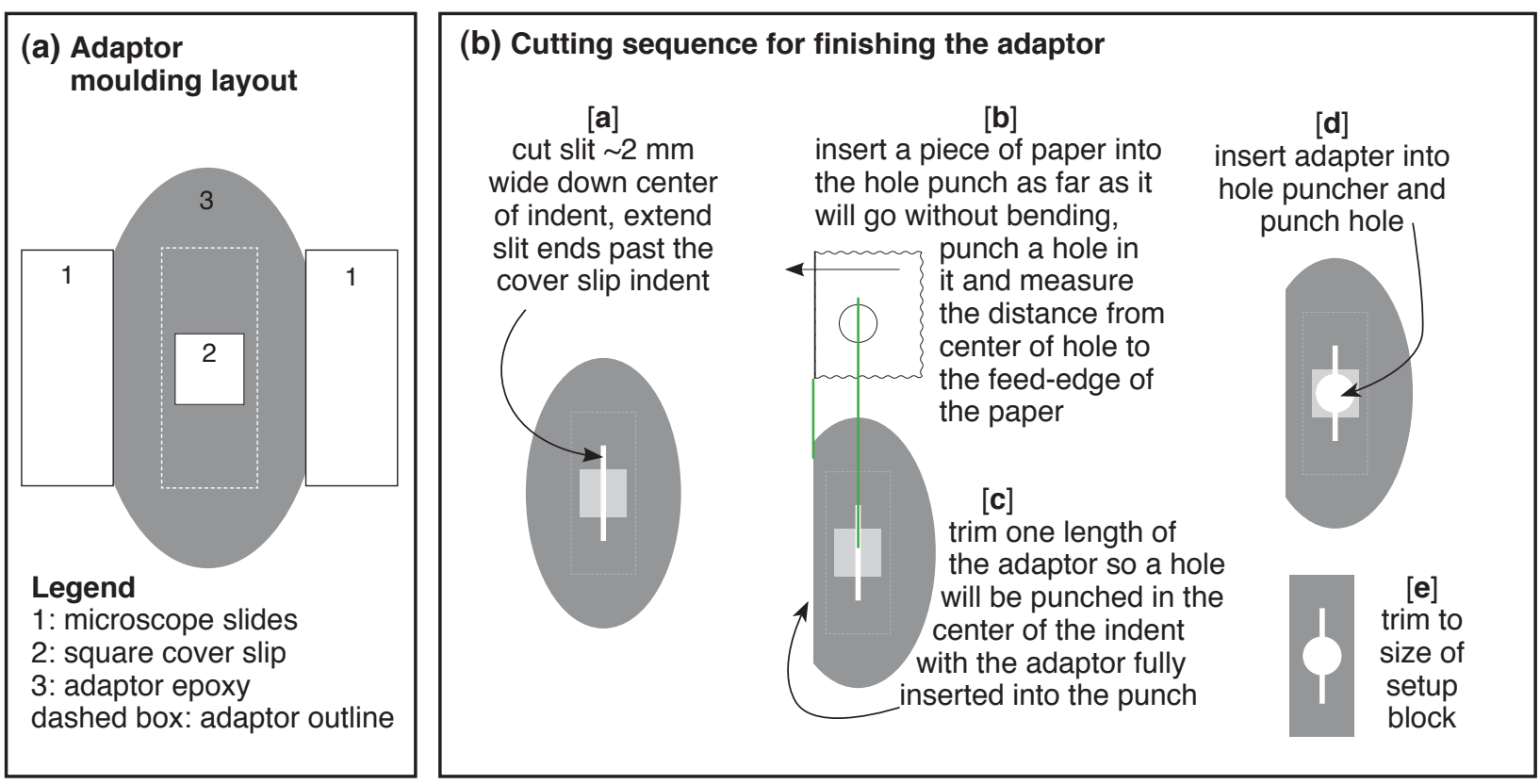

Figure S1: Forming the adaptor-spacer. (a) Adaptor moulding layout; (b) Cutting sequence for finishing the adaptor. 


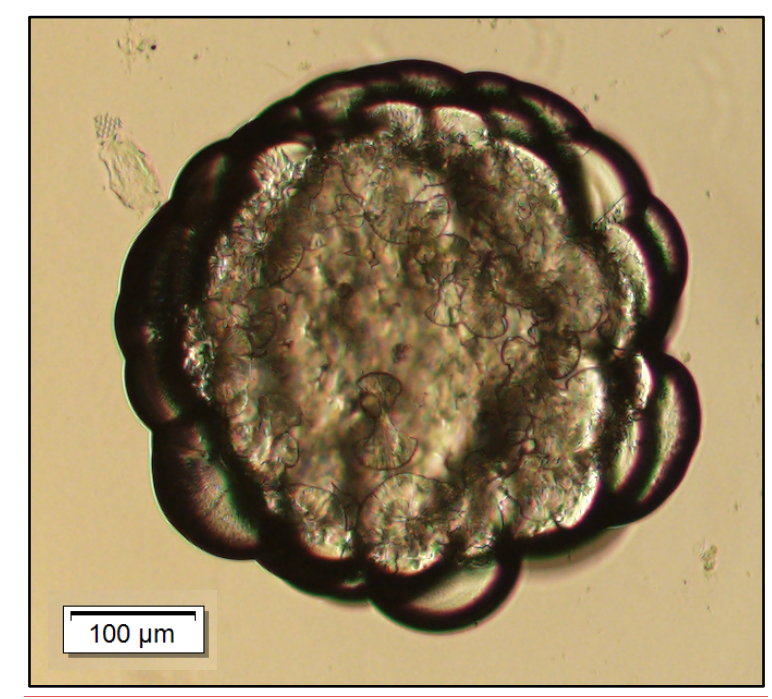

Figure S2: Photomicrographs of a complex object that precipitated in the diffusion gel of the described apparatus. Image taken on an Olympus IX inverted microscope with DP73 camera. Left image is overview of the precipitated object (scale bar $=100 \mathrm{um}$.) Internal details shown below (scale bar $=20$ um.)

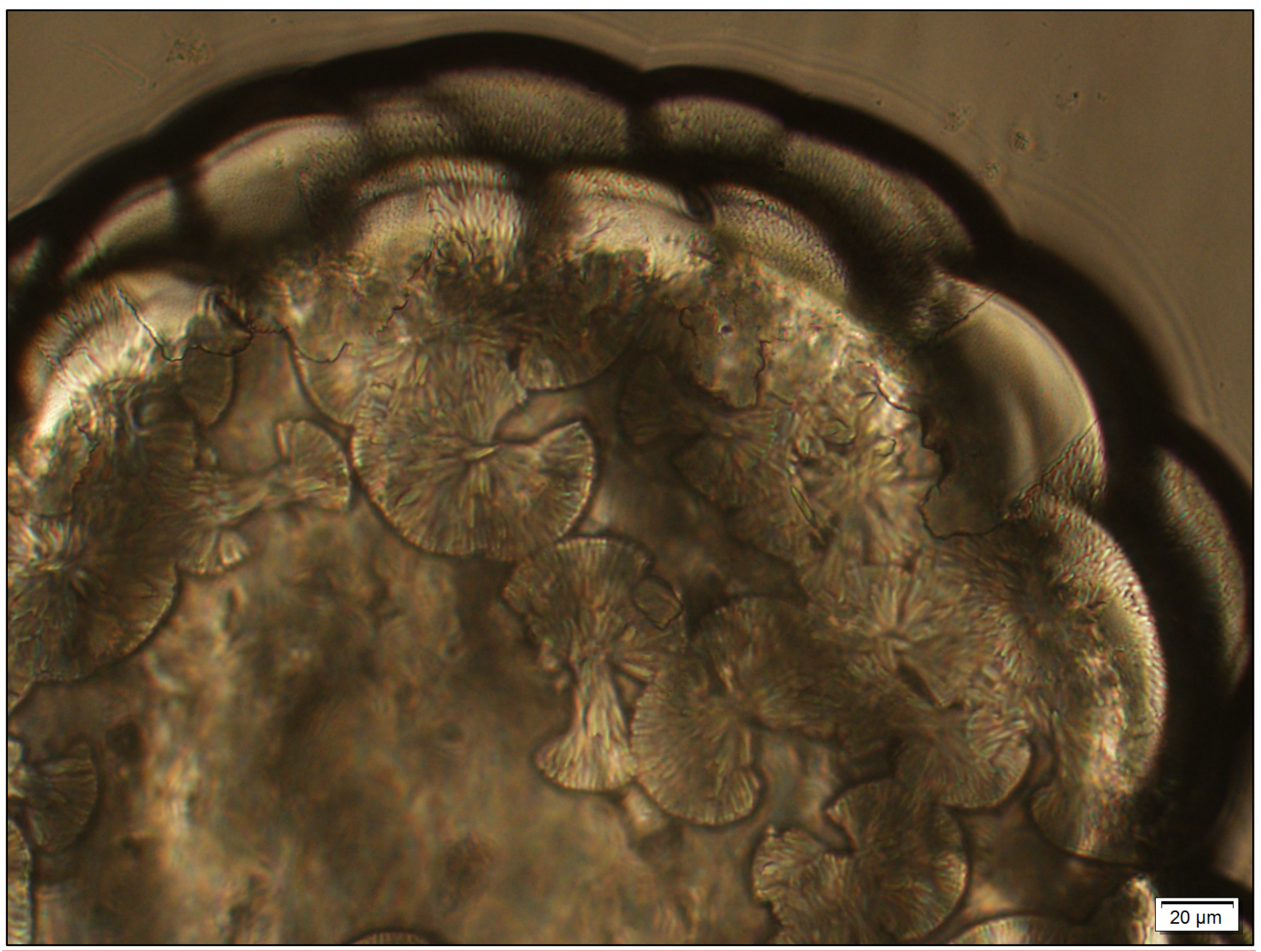

